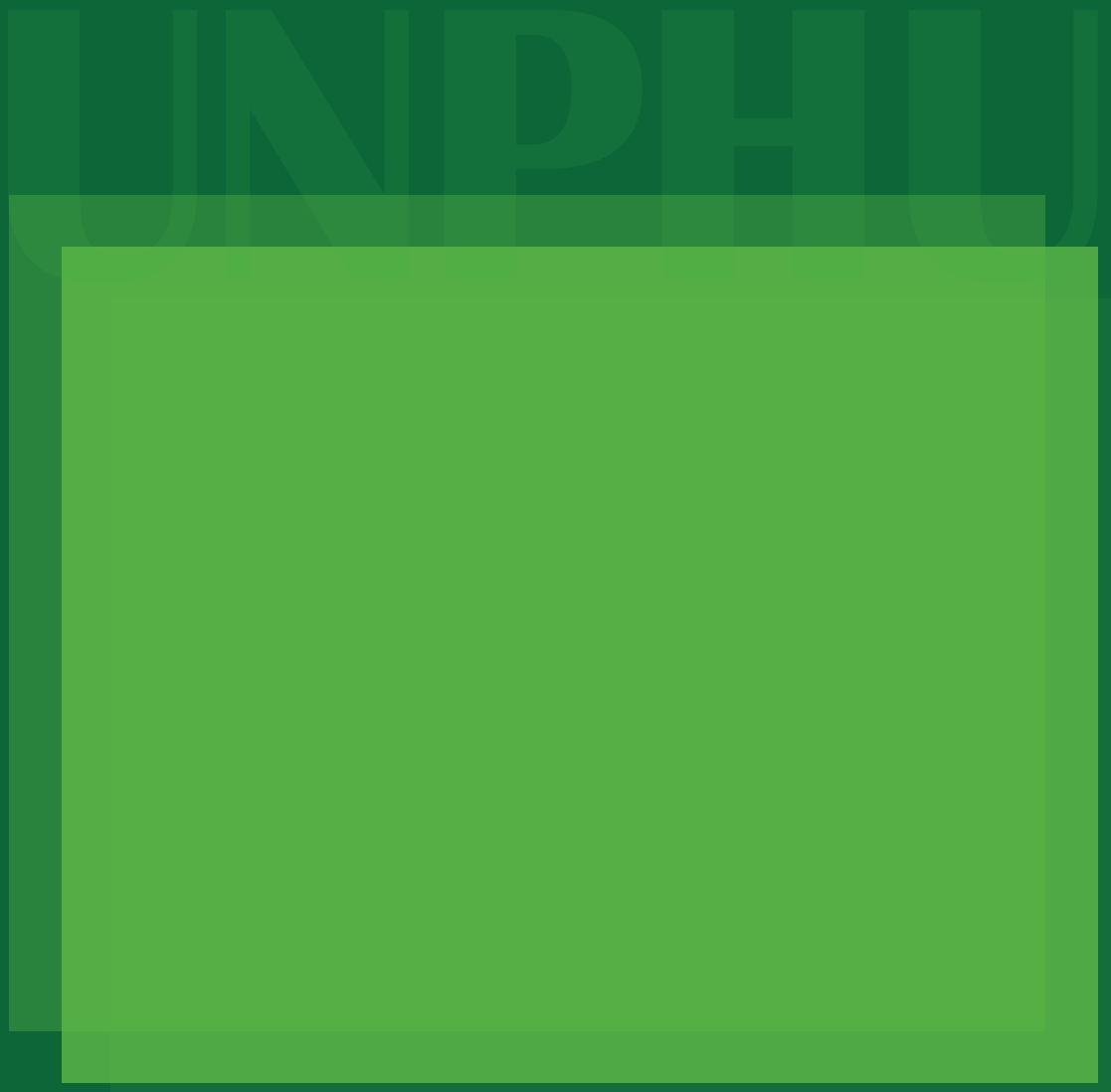




\section{Fenómenos climáticos extremos y sus efectos en el Caribe}

\section{Extreme Meteorological Phenomena and their Effects in the Caribbean}

Rafael Méndez Tejeda

Director del Dpto. de Ciencias Atmosféricas

Universidad de Puerto Rico, Carolina

rafael.mendez@upr.edu

Fecha de recepción: 18 de enero de 2018.

Fecha de aceptación: 3 de marzo de 2018

Favor de citar este artículo de la siguiente forma:

Méndez Tejeda, R. (2018). Fenómenos climáticos extremos y sus efectos en el Caribe.

Revista AULA. Vol. 61, Número 2, enero-junio 2018. Santo Domingo: Amigo del Hogar

\section{RESUMEN}

Durante los primeros quince años de este siglo, los fenómenos meteorológicos extremos (ciclones tropicales, olas de calor, frentes fríos, incendios forestales, sequías, etc.) han mostrado una tendencia que va en aumento. El propósito principal de este artículo es efectuar un análisis de los fenómenos meteorológicos extremos (FME), con el objetivo de motivar a la reflexión a los tomadores de decisiones y al público en general sobre la necesidad de estar preparados para los posibles efectos de estos eventos Además, el artículo analiza el impacto de estos fenómenos en los diferentes aspectos del diario vivir, tales como: salud de la población con enfermedades como dengue, chikungunya, zika, irritación de ojos y piel, entre otros; así como sobre la economía del país. En el caso particular de la República Dominicana, enclavada en el centro del Caribe y su dependencia del turismo y la agricultura, el preparar planes de mitigación y adaptación con el propósito de ser más resilientes es una obligación del Estado, así como de los gobiernos municipales.

Palabras clave: cambio climático, Caribe, clima, fenómenos meteorológicos extremos, República Dominicana

\section{ABSTRACT}

During the first 15 years of this century, Extreme Meteorological Events (tropical cyclones, heat waves, cold fronts, forest fires, droughts, etc.) have been a growing trend. The main purpose of this article is to carry out an analysis of Extreme Meteorological Events, with the aim of motivating decision-makers and the general public to reflect on the need to be prepared for the possible effects of these events. In addition, the article analyzes the impact of these phenomena on different aspects of daily life, such as: health of the population with diseases such as dengue, Chinkunguya, Zika, eye and skin irritation, among others, as well as in the economy of the country. In the particular case of the Dominican Republic, located in the center of the Caribbean and its dependence on tourism and agriculture, preparing mitigation and adaptation plans with the purpose of being more resilient is an obligation of the state, as well as of the municipal governments.

Keywords: Caribbean, climate, climate change, Dominican Republic, extreme meteorological phenomena 


\section{Fenómenos climáticos extremos y sus efectos en el Caribe Extreme Meteorological Phenomena and their Effects in the Caribbean}

\section{Introducción}

La presencia habitual en los medios de comunicación del tema (estado) del tiempo ${ }^{1}$ y el clima, ${ }^{2}$ ha despertado el interés del público y de la comunidad científica. Esto a pesar de que, durante mucho tiempo, el clima fue un gran desconocido. Por lo general, no nos enteramos de su importancia hasta que un fenómeno meteorológico nos arruina el día. Sin embargo, el clima es un actor principal en nuestras vidas, porque rige todas las actividades que realizamos; lo que comemos, lo que vestimos y hasta lo que tomamos depende de él. Por ejemplo: durante un día lluvioso tendemos a comer sopas o caldos, mientras que durante un día caluroso vestimos con ropa ligera, y durante un día soleado y caluroso nos vamos a la playa o a la piscina. Todos sabemos el impacto del clima en el comercio, entre otras cosas. La importancia del clima influye en el estado de ánimo de las personas, así como en la salud. Por lo tanto, conocer más sobre este tema será imprescindible, sobre todo debido la variabilidad climática como consecuencia del cambio climático y los eventos extremos asociados al calentamiento del planeta.

El carácter catastrófico de un fenómeno meteorológico no depende exclusivamente de cómo se manifieste, sino de la intensidad con que impacte la variable (temperatura, humedad, precipitación, viento, etc.). Las particularidades geomorfológicas de la zona afectada, la distribución de la población, los usos del terreno, y las prácticas inadecuadas e intervenciones humanas en el territorio, juegan un papel esencial en una catástrofe. La ocupación de los cauces, las llanuras de inundación y los conos de deyección de los torrentes de agua, y los motivos económicos o sociales pueden condicionar el carácter catastrófico de un fenómeno extremo de cierta habitualidad.
La Organización Meteorológica Mundial (OMM), define "Fenómenos Meteorológicos Extremos (FME)" como un episodio, suceso o evento meteorológico que es raro o infrecuente, según su distribución estadística para un lugar determinado. En la actualidad, la humanidad está experimentando una serie de FM E (huracanes, tormentas, lluvias intensas, nevada, olas de calor y de frio, sequias, entre otros) que ocurren en un mundo diferente del que conocieron nuestros abuelos. La temperatura media de la atmósfera ha aumentado $0.75^{\circ} \mathrm{C}$ en apenas medio siglo. Los océanos están acumulando más calor y por lo tanto aumenta su temperatura, mientras que en los polos el hielo se está derritiendo a un ritmo acelerado, al igual que muchos glaciares en las montañas. Los episodios de lluvia, nieve intensa, incendios forestales, deslizamientos de tierra, entre otros, se han vuelto más frecuentes y los períodos secos son cada vez más prolongados. El aumento de estos fenómenos climáticos está afectando grandemente la flora y la fauna de todo el planeta y muy severamente la región tropical.

Para calcular la frecuencia de un FME se debe estudiar el comportamiento de estos fenómenos. Se utilizan las series de observación y se determina cuál es la probabilidad de aparición de la situación meteorológica que dio lugar a un desastre natural. Las series de valores extremos que se utilizan, muchas de ellas se remontan al siglo pasado, presentan en general, una homogeneidad probada. Si se observara realmente una mayor frecuencia de aparición de sucesos extremos se tendría que pensar en una alteración de la estabilidad y la ciclicidad climática, ya que con observar simplemente las series disponibles de datos no se desprende esta conclusión claramente, sino del análisis profundo y bien ponderado. Los cambios climáticos, siempre han sido parte de la evolución del planeta Tierra, pero la celeridad de estos cambios y el aumento en la 
frecuencia e intensidad de los FME son el resultado del aumento de concentraciones de gases invernadero a partir de la revolución industrial, tales como: el dióxido de carbono, metano, vapor de agua, óxidos nitrosos y clorofluorocarbonos en la atmósfera terrestre; produciendo un aumento abrupto de la temperatura promedio terrestre, esto es lo que se conoce como calentamiento global.

Es importante señalar la relación entre que existe entre el aumento en la temperatura de los océanos y los mares tropicales, donde se produce una mayor evaporación, debido a que el trópico es la región del planeta que recibe mayor cantidad de radiación solar durante el año. Esto genera una mayor cantidad de nubosidad, la cual al ser integrada en la atmósfera, produce células de circulación atmosférica en toda la primera capa de la atmósfera (tropósfera). Esta nubosidad producirá en los trópicos y latitudes medias, lluvias de mucha intensidad y corta duración. Sin embargo, en las altas latitudes (USA, Canadá, Europa, etc..) se precipitará en forma de nieve, causando frentes fríos y grandes nevadas por lo que los inviernos tenderán a ser más intensos y los veranos cada vez más cálidos, de acuerdo con los pronósticos de la Organización Meteorológica Mundial (OMM). Estos frentes fríos han causado y están causando un gran impacto en la erosión costera de todo el Caribe. Ortiz-Royero, et al (2013).

En el momento en que se escribe este artículo, en Estados Unidos y Europa se están registrado olas de fríos intensas. Sin embargo, al analizar los registros de la tendencia de la temperatura en EU. UU., a partir de mediados del siglo pasado, muestran que las temperaturas máximas están cambiando con una frecuencia de más del doble que los récords de temperaturas mínimas y se han observado patrones similares en todo el planeta. En el año 1950 la relación entre días de calor y días de fríos era de un día de calor por cada 1.09 día de frio (1: 1.09), ya en el año 2000 la relación ha cambiado totalmente. Meehl et al
(2009) reporta que por cada día de frío tenemos 2.04 día de calor (1: 2.04), esta tendencia aumentaría si el análisis se extiende al 2018.

Al analizar los bancos de datos de la Administración Nacional de Atmósfera y Océano (del acrónimo en inglés NOAA), desde 1880, en que se tienen registros históricos instrumentales, los diez años más cálidos corresponden al siglo XXI, siendo el 2015 el año más cálido de ese registro. Esta Organización, después de haber reunido los principales conjuntos de datos internacionales, observó que la diferencia de temperatura entre los años más cálidos era tan solo de varias centésimas de grado, o sea, menor que el margen de incertidumbre.

Desde la década de 1990 los pronósticos han planteado diversos escenarios sobre cómo cambiaría el clima del planeta, pero todos o casi todos han coincidido en que los FEM van a aumentar en frecuencia e intensidad, por lo que es urgentela preparación para estos nuevos escenarios. Por otro lado, también se han visto afectadas algunas anomalías climáticas estacionales en numerosas partes del mundo, tales como la Niña o el Niño, aunque resulta difícil atribuir explícitamente la causa de un fenómeno meteorológico extremo a El Niño o La Niña sin tener en cuenta la influencia de otros factores. En la actualidad se están llevando a cabo nuevas investigaciones para establecer de manera concluyente si el cambio climático provocará episodios más frecuentes e intensos de El Niño y/o La Niña. Tartaglione, (2003)

Algunos investigadores tales como Schuldt, (2016) and Woosuk et al (2017) entre otros, han encontrado que en los quince años de este siglo la frecuencia y la intensidad de estos eventos se ha incrementado respecto a las últimas décadas del siglo XX; la relación entre intensidad de estos fenómenos extremos de acuerdo con sus hallazgos es sólida. Se estima que los océanos han absorbido alrededor de veinte veces más calor que la atmósfera durante el último medio siglo, provocando temperaturas más altas en aguas superficiales y profundas. Así ha 
sido confirmado por otros investigadores, tales como: IPCC (2016) and NCA (2014). El tener una temperatura de los océanos más alta, es sinónimo de tener mayor combustible (índice calor acumulado) para la formación de ciclones tropicales de mayor intensidad. UCS (2017) sustenta la hipótesis que existe una relación positiva entre la intensidad de los ciclones tropicales y la temperatura superficial de los océanos.

\section{Aumento en la temperatura}

De acuerdo al IPCC 2007 de los doce últimos años (1995-2006), once figuran entre los doce más cálidos en los registros instrumentales de la temperatura de la superficie mundial (desde 1850). La tendencia lineal a 100 años (1906-2005), es de $0,74^{\circ} \mathrm{C}$, es decir para la temperatura mínima es de $0,56^{\circ} \mathrm{C}$ y para la temperatura máxima $0,92^{\circ} \mathrm{C}$, esto es superior a la presentada en el informe anterior presentado por mismo IPCC que es $0,6^{\circ} \mathrm{C}$ [entre $0,4^{\circ} \mathrm{C}$ y $0,8^{\circ} \mathrm{C}$ ] para el periodo $(1901-2000)$. Este aumento de temperatura está distribuido por todo el planeta y es más acentuado en las latitudes septentrionales superiores. Las regiones terrestres se han calentado más aprisa que los océanos.

Para la región del Caribe, algunos investigadores como Stephenson et al (2014) han reportado una tendencia en los índices de temperatura y precipitación diarios y extremos en la región del Caribe para los registros que abarcan los intervalos de 1961-2010 y 1986-2010. Méndez-Tejeda (2017) encuentra para Puerto Rico un aumento en la cantidad de días de calor por décadas, los cuales se han triplicado entre la década de 1960 hasta década del 2000. También reporta que la temperatura ha aumentado la cantidad de $2.24 \mathrm{C}$ en los últimos 60 años. Por otro lado, usando modelo de circulación global Van Beusekom et al (2014), Jury (2010) y Comarazamy ( 2011) ha reportado un aumento $0.01^{\circ} \mathrm{C} /$ año a $0.03^{\circ} \mathrm{C}$, lo que representa un aumento entre $1^{\circ}$ a $3^{\circ} \mathrm{C}$ en 100 años.

\section{Aumento en los incendios forestales}

Los incendios forestales son un tipo de fuego que se extiende sin control sobre el terreno, afectando la vegetación y todo el entorno. Estos constituyen una grave amenaza para el medio ambiente; sin embargo, cada año, miles de hectáreas son afectadas por los incendios forestales. Las sequías, que se han hecho cada vez más frecuentes, son un gran aliado para que este fenómeno se expanda. Diversos países como España, Australia, Chile, EE. UU. y los que pertenecen a la región del Caribe, entre otros; han experimentado este incremento en los incendios forestales. Méndez Tejeda el al (2015), evalúa el impacto económico que representa este fenómeno extremo para Puerto Rico. Entre los hallazgos está la contribución que hace la sequía creando condiciones óptimas para que estos incendios se propaguen. Este mismo artículo reporta que la mayoría de estos incendios son de origen antropogénico; es decir; con intervención del ser humano, aunque el cambio climático haya creado las condiciones necesarias para su propagación.

En el caso de California (EE. UU.), los incendios forestales han comenzado a ser fenómenos habituales cada año, dejando grandes pérdidas de vidas y bienes. En la República Dominicana en los años recientes, la provincia de Montecristi, Valle Nuevo en la Cordillera Central, Azua en el sur, entre otros, han tenido ocurrencia de incendios forestales al igual que en Puerto Rico, donde la mayoría son provocados, aunque el factor meteorológico crea las condiciones idóneas para su rápida propagación

\section{Aumento del nivel del mar}

La comunidad científica está de acuerdo que para el 2100 el nivel del mar debe haber subido por lo menos, 1.2 metros sobre el actual. No será de una forma lineal sino cuasi-exponencial, debido a las retroalimentaciones, así como por factores de índoles meteorológicas y astro- 
nómicas. Pero de cualquier forma este aumento está impactando las playas de todo el mundo y, muy especialmente, en la región del Caribe. Estos efectos se pueden observar en las siguientes fotografías:

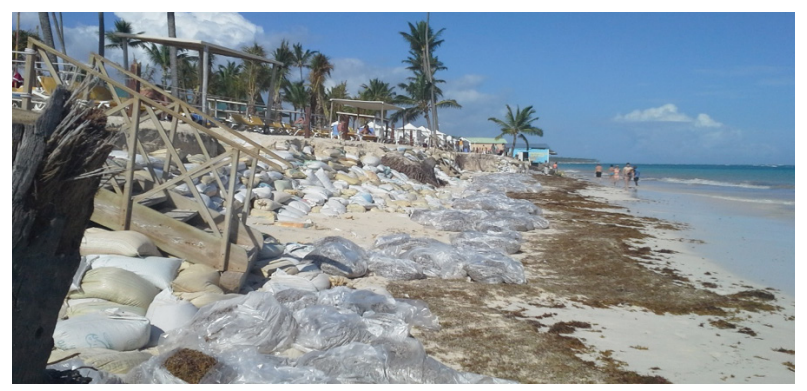

Fig. 1. Muestra la erosión en las playas de la región este de la República Dominicana en el mes de febrero de 2016.

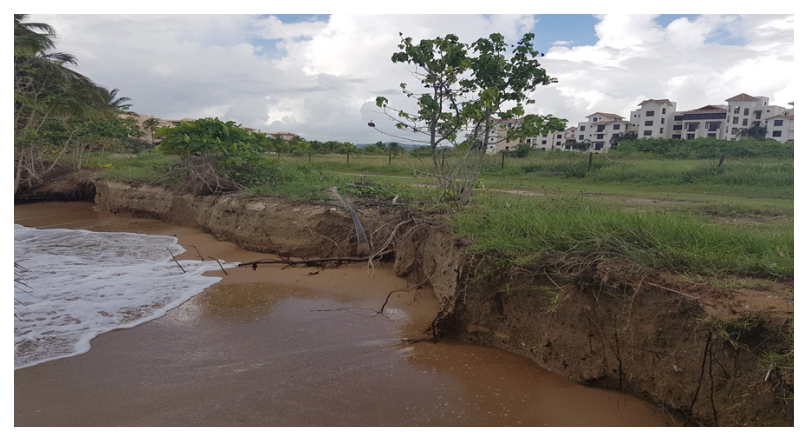

Fig. II. Muestra la erosión en las playas de la región este de Puerto Rico en el mes de febrero de 2016.
Entre las causas principales que han producido cambios morfológicos en las playas y costas del Caribe durante las últimas décadas se pueden mencionar:

1) La ocurrencia de marejadas (producidas por sistemas ciclónicos, sistemas frontales, frentes fríos, etc.);

2) La variabilidad en la descarga de agua y sedimentos de los ríos;

3) El impacto en las barreras naturales (corales, arrecifes, praderas de yerbas marinas y roca de playa) sobre las costas;

4) La presencia de cañones submarinos y

5) Modificación de las desembocaduras de los ríos, arroyos y humedales.

Todas estas variables pueden promover pérdida o ganancia de sedimentos en los sistemas de playa, según Barreto, (1997) and Morelock (1984). Por otro lado, algunos estudios realizados han identificado que diversas actividades humanas, tales como la extracción de arena de las dunas, los dragados en la zona litoral, las construcciones de infraestructura en la costa, los cambios de uso y cobertura de terrenos, así como la alteración de cuencas hidrográficas y la presencia de represas en ellas, pueden mo-

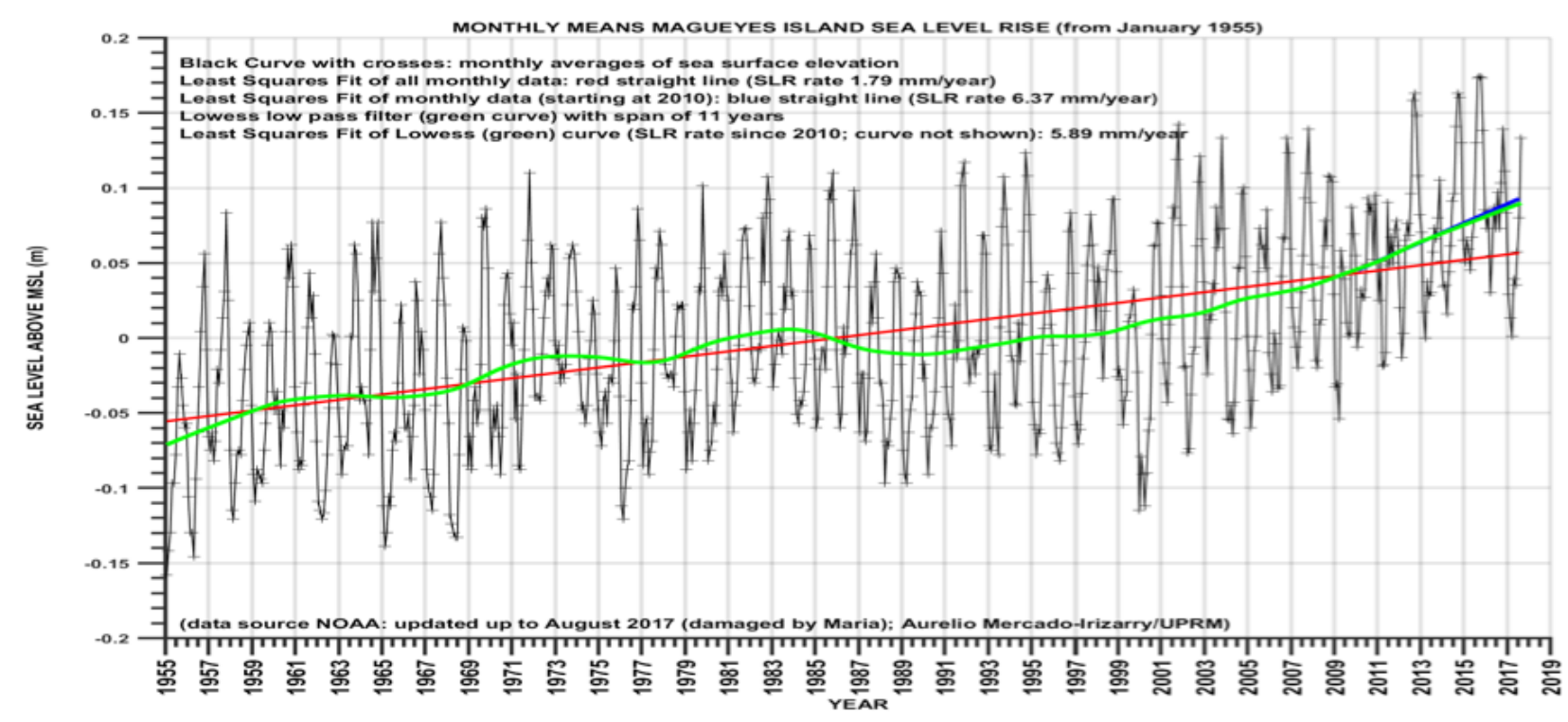

Fig.3.- Se observa el mareógrafo colocado en La Perguera, Puerto Rico, para el periodo de 1955 hasta 2017. (Cortesía del Dr. Aurelio Mercado) 
dificar la morfología de los sistemas de playas French and Burningham (2013).

En la gráfica anterior, puede observarse el mareógrafo colocado en La Perguera Puerto Rico, apenas a $80 \mathrm{~km}$ de la costa de República Dominicana. Podemos ver, en la figura, que el aumento en el nivel del mar ha ocurrido desde que se lleva récord (curvas negras), aunque no de una manera constante. Las curvas azules en gráficas muestran una media móvil (running average) de diez años, que tiende a suprimir variaciones de una década o menos. Es un suavizamiento de los datos que muestra la variabilidad a largo plazo. Este mismo comportamiento debe esperarse para las costas del este de la República Dominicana, donde lamentablemente se carece de bancos de datos fiables.

\section{Inundaciones}

El aumento de las lluvias intensas a partir de las últimas dos décadas es un reto que tienen que superar los meteorólogos. Mejorar la capacidad de predicción temprana de los FME y buscar mecanismos para adaptarse a los cambios, son dos de los grandes desafíos a enfrentar ante el cambio climático.

Podemos definir una lluvia intensa cuando en 24 horas recibimos una precipitación superior a la $50.8 \mathrm{~mm}$, por lo general estas lluvias son de origen convectivo, es decir, que no son lluvias que provienen de sistemas ciclónicos Groisman et al (2005), sino que su origen mayormente es producido por el calor diurno y la orografía del terreno. Estas lluvias son más frecuentes en las ciudades costeras donde la brisa juega un rol importante, así como la isla de calor ${ }^{3}$, producidas por las edificaciones y el pavimento. Estas inundaciones son repentinas y causan grandes problemas en el transporte de las ciudades. Por ejemplo, los autos pueden perder el control o estancarse. El agua puede arras- trar lodo y basura que pueden contener aguas negras y químicos causando enfermedades en los seres humanos y animales, estos escombros en las aguas de inundaciones pueden ocasionar heridas a los seres humanos, en definitiva, causar daños a vidas y propiedades.

\section{Sequías}

La sequía puede ser considerada como una amenaza climática que inicia con una disminución acusada de la precipitación promedio, ocasionando escasez del recurso agua en un lugar determinado durante un periodo específico. Generalmente, si la lluvia es $75 \%$ o menos de lo normal (lluvia promedio de 30 años) por un periodo de un año o más, se considera una sequía meteorológica. Debido a la variabilidad natural del clima, la probabilidad de ocurrencia y frecuencia de estos episodios meteorológicos es cada vez mayor, lo cual afecta la vida de muchas personas y ocasiona pérdidas económicas en un país. En la mayoría de los casos, la presión que ejerce la escasez del recurso hídrico (la sequía) sobre el sistema se traduce en sequías agrícolas, sequías hidrológicas y sequía social. Magaña and Neri (2007)

Por otro lado, definimos la sequía agrícola ocurre cuando no hay suficiente agua para que puedan crecer los cultivos, afectando mayormente la producción de cosechas y el ganado. Mientras que la sequía hidrológica se refiere al efecto de la merma en el patrón de lluvia por un periodo prolongado sobre los cuerpos de agua superficiales y sobre los recursos de agua subterráneos y los efectos de esta escasez en el suministro de agua. Por último, pero no menos importante, la sequía social que está relacionada con los efectos de la escasez en el abasto de agua para las personas y las actividades económicas, potenciando desigualdades de acceso y aprovechamiento del agua en la población del país. La sequía más reciente ocurrida en el 
Caribe fue entre los años 2013 a 2015, ocasionando diversas pérdidas económicas en toda la región, la que coincidió con la presencia del fenómeno de El Niño. NWS (2016)

\section{Impactos en la salud}

Recientemente Méndez-Lázaro et (2015), reportaron que el calentamiento global afecta la salud de la población en el Caribe y causa un aumento de las tasas de mortalidad y de morbilidad. También incrementa la vulnerabilidad de la población. Los grupos de edad de alto riesgo, como las personas de edad avanzada y niños no están preparadas para resistir temperaturas mucho más altas. Si se suman el calentamiento global y las islas urbanas de calor, las temperaturas podrían ascender hasta seis grados Celsius por encima de lo normal. En el Caribe, se están registrando temperaturas altas extremas con mayor frecuencia que nunca. Méndez Tejeda et al (2017) and Rhiney, (2015).

Se puede asociar una mayor frecuencia de enfermedades respiratorias y de cáncer en la piel con las condiciones creadas por el cambio global. Por ejemplo, el aumento en la prevalencia de asma, cáncer de la piel y de mayor incidencia de dengue en la población de Puerto Rico puede relacionarse con los efectos del cambio climático. La cantidad de ozono presente en nuestra tropósfera, combinado con una mayor cantidad de partículas en suspensión, como el polvo del Sahara o las cenizas del volcán Le Soufrière, en Montserrat, entre otros contaminantes, ha incrementado la cantidad de casos por afecciones respiratorias Méndez-Lázaro et (2016) and NCA (2016). A largo plazo, tratar todos estos casos conlleva un costo y una inversión mayor por parte de la ciudadanía y del gobierno.

La relación entre la salud y el clima ha sido estudiada desde Hipócrates, el padre de la Me- dicina en una publicación titulada "Agua, aire y lugares", dice textualmente: "Quien desee estudiar correctamente la ciencia de la medicina deberá proceder de la siguiente manera. Primero, deberá considerar qué efectos puede producir cada estación del año, puesto que las estaciones no son todas iguales, sino que difieren ampliamente tanto en sí misma como en sus cambios. En el tratado hace también referencia a los vientos cálidos y a los fríos, entre otros fenómenos climáticos."

En la actualidad con el aumento en la tendencia de los FME, se hace más necesario que nunca, estudiar ampliamente estos vínculos, oscilación del sur y sus dos fases; la fase fría (La Niña) y su fase cálida (El Niño), están íntimamente relacionadas con la intensificación de enfermedades transmitidas por insectos, en especial el mosquito Aedes aegypti, vector que transmite dengue, zika y chikunguya. Seguinot (2005) and Rigau-Pérez, et al (2002).

Recientemente, Méndez-Lázaro et al (2016,) han encontrado para Puerto Rico una relación entre las olas de calor y un aumento en las enfermedades respiratorias, reportando que se han duplicado las muertes cuando la temperatura se mantiene entre $33^{\circ} \mathrm{C}$ y $38^{\circ} \mathrm{C}$. Resultados similares han sido mostrados por Burkard et al (2010), para la región de Texas y México.

Otro FME que se ha intensificado en a partir de 2003 de acuerdo con hallazgos de Jiménez-Vélez et al (2009), es la presencia del polvo del Sahara y el aumento de los hongos y esporas que impactan la salud grandemente. De esta forma influye en el asma bronquial, alergias, afecciones de la piel y dolencias cardiovasculares. La llegada cada año a la región del Caribe de grandes cantidades de este particulado en forma de nubes generadas por las tormentas de arena del desierto del Sahara, también tienen un enorme impacto en los ecosistemas marinos y 
terrestres. Anualmente se forman enormes tormentas sobre el desierto del Sahara y la región conocida como el Sahel en el norte de África, las cuales incorporan millones de toneladas de polvo a la atmósfera. Estas nubes de polvo contienen partículas muy finas, o aerosoles, que por su pequeño tamaño pueden permanecer suspendidas en la atmósfera y transportarse mediante los vientos alisios a regiones distantes como Europa, el Medio Oriente y el oeste del océano Atlántico Detrés (2015).

Aunque no sabemos exactamente cómo cambiará la distribución de las temperaturas, la mayoría de los climatólogos coinciden en que, en promedio, el clima mundial seguirá calentándose IPCC (2014). No obstante, para un meteorólogo se hace más difícil hacer una predicción a corto plazo que a largo plazo, por tal razón tenemos más incertidumbre en los pronósticos a corto plazo. Aquí es donde un juega un papel determinante la climatología en la que las predicciones se hacen a largo plazo, por lo que su grado de certidumbre es mucho mayor. Basándonos en la mayor certidumbre de la climatología nos atrevemos a pronosticar que tendremos en el futuro cercano un aumento en la frecuencia de fenómenos climáticos extremos y de mayor intensidad. Corresponde a la sociedad y a los gobiernos hacer política pública sobre adaptación, mitigación y resiliencia con el objetivo de minimizar los impactos que estos fenómenos ocasionarán en los años próximos.

\section{Ciclones tropicales}

Desde mediados de la década de 1980, coincidiendo con el aumento de la temperatura media del planeta Tierra, la frecuencia y la intensidad de los huracanes ha ido en aumento. Wei et al (2016) Pielke (2008) and UCS (2017). Desde 1998, con el impacto del huracán George, hasta el Huracán María del 2017, el océano Atlánti- co ha sido escenario del desarrollo de diversos ciclones y tormentas tropicales. El 2005, año récord, en la actividad ciclónica se forman 28 sistemas que causaron enormes daños en el Caribe y en los USA.

Durante el año 2017, los huracanes que han azotado Estados Unidos y el Caribe tendrán fuertes repercusiones económicas y sociales. A los elevados costos de reconstrucción hay que agregar el impacto sobre el turismo, el mercado de seguros y hasta una reducción en las estimaciones de crecimiento de Estados Unidos. La actual temporada de huracanes con Harvey, Irma y María (por mencionar los de mayor categoría), se ha convertido en una de las más costosas de la historia. Además de los cientos de muertos, heridos y damnificados que los huracanes han dejado a su paso por el Caribe y Estados Unidos, los costos económicos son enormes.

La agencia clasificadora de crédito Moody`s estimó inicialmente los daños causado por Harvey para el sureste de Texas entre 50 y 75 billones, clasificándola como una de las tormentas más costosas de la historia de Estados Unidos. Junto a catástrofes como el huracán Katrina en 2005 que costó 81 billones y el huracán Sandy en 2012 que arrojó pérdidas por 35 billones. En el caso del Huracán María el impacto en Puerto Rico superó los 94 billones de dólares.

En cuanto al turismo, en el 2016 los turistas gastaron 31 billones de dólares en el Caribe y se esperaba que estas cifras crecieran un 5.4\% para el 2017. Este crecimiento se ha visto afectado por los impactos de los ciclones tropicales, causando grandes pérdidas a la economía de la región del Caribe.

El comportamiento tanto del anticiclón de las Azores, como la gran cantidad de energía calorífica almacenada en el océano, que es en- 


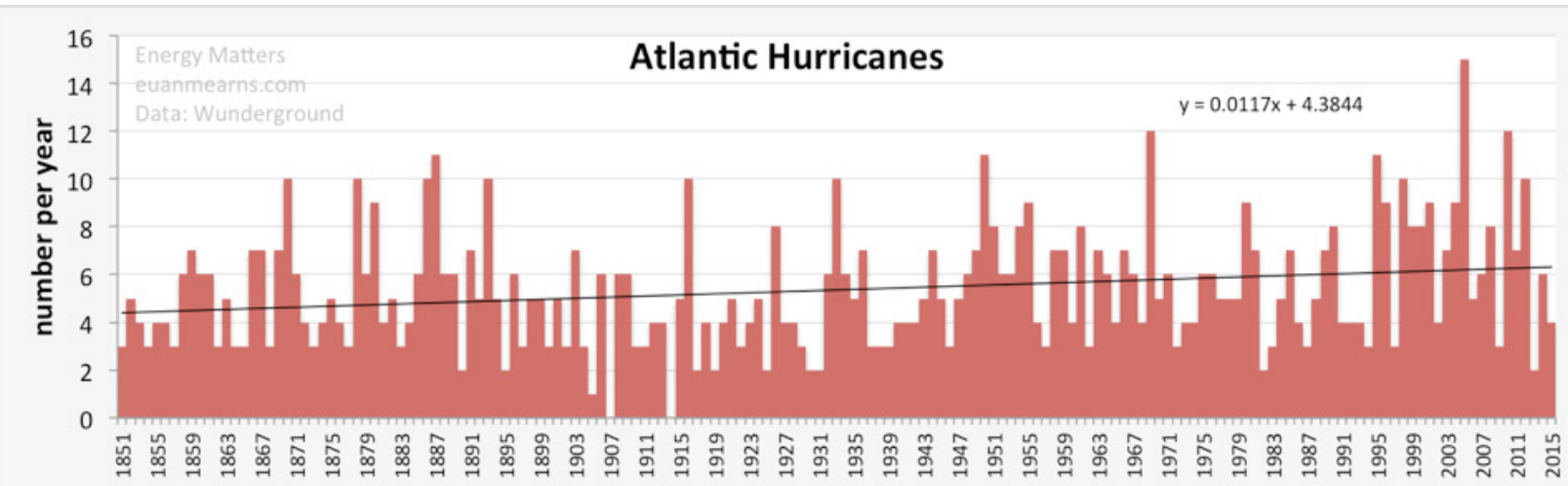

Fig. 4.- Muestra el comportamiento de los huracanes en el Océano Atlántico en el periodo 1950 hasta el 2015. En él puede observarse una tendencia positiva a través de todo el periodo. (fuente NOAA)

viada a la atmósfera, así como la ausencia del fenómeno de El Niño y el posible desarrollo de La Niña; son factores que pueden contribuir a una temporada de huracanes muy activa en el 2018. Normalmente, la presencia de El Niño nos trae una corriente de aire cortante en la troposfera que tiende a debilitar los huracanes en el océano Atlántico. Por tal razón, la población del Caribe debe prepararse para mitigar los impactos que pueda generar la próxima temporada ciclónica.

\section{Factor de riesgo vulnerabilidad}

El cambio climático hace que las hipótesis relativas a la frecuencia y gravedad de las amenazas climáticas derivadas de la experiencia histórica dejen de ser una base fiable para la evaluación de riesgos a corto plazo. Si bien es cierto que la conciencia acerca de los riesgos climáticos ha aumentado notablemente, a menudo las instituciones nacionales no están lo suficientemente preparadas para responder y prevenir los riesgos asociados a las nuevas y múltiples amenazas que afectan a distintos sectores. Esto se suma a una falta de claridad sobre mandatos y distribución del trabajo entre los distintos organismos y departamentos que se reparten las responsabilidades de la gestión de los riesgos de desastre.
La gestión del riesgo climático se centra en el desarrollo de sectores que, como la agricultura, los recursos hídricos, la seguridad alimentaria, la salud, el medio ambiente y los medios de subsistencia; son muy sensibles al cambio y a la variabilidad del clima. Para el Programas de las Naciones Unidas para el Desarrollo (Acrónimo en inglés del PNUD), la gestión y la prevención de los riesgos climáticos implica no sólo el replanteamiento de las vías de desarrollo, las políticas y los marcos institucionales tradicionales, sino también el fortalecimiento de las capacidades locales, nacionales y regionales para diseñar e implementar medidas de gestión de riesgos, mediante la coordinación de una amplia gama de actores, entre los que se encuentran el sistema de la ONU, los gobiernos nacionales, organizaciones no gubernamentales, organizaciones de la sociedad civil y miembros de la comunidad científica.

La mitigación y resiliencia de un país, está íntimamente ligada al nivel de preparación del país. Por lo tanto, se deben adoptar medidas que apunten a la adaptación de las economías, recursos y población, a las vulnerabilidades particulares que se presenten en cada región o país. En el caso de la República Dominicana, los principales desafíos para crear capacidades de resiliencia económica, social, sanitaria y cultural de las personas, las comunidades, sus bienes, así como del medio ambiente en una 
necesidad imperiosa. Por tal razón, esto representa un reto importante para el gobierno y el sector privado, que de no enfrentarse, los avances logrados en las últimas dos décadas pueden perderse en un día. "Please", ver impacto del huracán María en Puerto Rico.

\section{Agradecimientos}

A la Dra. María Santos-Corrada (Universidad del Turabo), al Dr. Carlos Severino (UPR Rio Piedras) y al Dr. Miguel Ángel Fornerin (UPR Cayey) por sus importantes sugerencias.

\section{Notas}

1. Definimos Tiempo como las condiciones meteorológicas que describen el estado de la atmósfera en un momento dado para un lugar específico. Ejemplo: el día de hoy es soleado, el tiempo mañana será caluroso, etc.

2. El Clima es la medida del Tiempo promedio para un lugar en particular. Se define por lo general para un mes o una estación del año, y considera el promedio de los datos del tiempo de 30 años. Ejemplo, Santo Domingo es una cuidad calurosa.

3. Isla de calor urbana es el término que se usa para describir el calor característico tanto de la atmósfera como de las superficies en las áreas urbanas (ciudades o pueblos) comparadas con sus entornos no urbanizados (zonas rurales).

\section{Referencias}

Barrera, R. (2010). “Dinámica del Dengue y Aedes Aegypti en Puerto Rico". Revista Biomédica ,Vol. 21, pp. 179-195
Barreto, M. (1997). Shoreline Changes in Puerto Rico [Cambios en la costa en Puerto Rico]. (1936-1993). Ph.D., University of Puerto Rico.

Brunkard, J.M., Cifuentes, E. y Rothenberg, S.J. (2008). Assessing the roles of temperature, precipitation, and ENSO in dengue re-emergence on the Texas-Mexico border region. [Evaluando los roles de temperatura, precipitación y oscilación sureña de El Niño (ENSO), en la reaparición del dengue en Texas, Méjico]. $\mathrm{Sa}$ lud Pública Mex., Vol. 50, pp. 227-234.

Burke, D. et al. (2001). Under the weather: Climate, ecosystems and infectious disease. [Clima, ecosistemas y enfermedades infecciosas]. Emerg Inf. Dis; 7: 66-608.

Comarazamy, D. E., González, J. E., Luvall, J. C., Rickman, D. L., \& Mulero, P. J. (2010). A land-atmospheric interaction study in the coastal tropical city of San Juan, Puerto Rico. Earth Interact. [Estudio de interacción atmosférica terrestre en la ciudad costera tropical de San Juan, Puerto Rico. Interactuar con la tierra.],Vol.14, pp. 1-24, http://dx.doi. org/10.1175/2010EI309.1

Detrés, Y. (2015). El polvo del Sahara y sus interacciones con el clima http://www. corrienteverde.com/articulos/el\%20 polvo $\% 20 \mathrm{del} \% 20$ Sahara $\% 20 \mathrm{y} \% 20$ sus $\% 20$ interacciones $\% 20$ con $\% 20$ el\%20clima.html

Douglas, G. (2014). The Neglected Climatic Hazards of the Caribbean: Overview and Prospects in a Warmer Climate. [Los peligros climáticos abandonados del Caribe: visión general y perspectivas en un clima más cálido.] Geography Compass 8:4, pp. 221-234. Online publication date 8 april 2014. 
Easterling, D. R., et al. (1997). Maximum and minimum temperature trends for the globe. [Tendencias de temperatura máxima y mínima para el globo terráqueo]. Science, p. 277, pp. 364-367.

French J.R. and Burningham H. (2013). Coasts and climate: Insights from geomorphology. Progress in Physical Geography [Costas y climas: perspectivas de la geomorfología]. 37(4) pp. 550-561. DOI: $10.1177 / 0309133313494962$

Groisman, P., Knigh, W., Easterling, D., Thomas, K.,Nicholas, H. y Razuvaev, V. (2005). Trends in Intense Precipitation in the Climate Record. Journal Climate. Vol. 18 pp. 1326-1350.http://journals.ametsoc. org/doi/abs/10.1175/JCLI3339.1

Groisman, P. et al. (1999). Changes in the probabilities of heavy precipitation: [Cambios en las probabilidades de precipitaciones fuertes]. Important indicators of climatic change, Clim. Change,Vol. 42, pp. 243-283.

Hall, T. C., Sealy, A. M., Stephenson, T. S., Kusunoki, S., Taylor, M. A., Chen, A. A., y Kitoh, A. (2013). Future climate of the Caribbean from a super-high-resolution atmospheric general circulation model. [Clima futuro del Caribe a partir de un modelo de circulación general atmosférico de alta resolución]. Theoretical and applied climatology, 113(1-2), 271-287.

Hansen J., Sato, M. y Ruedy, R. (2012).

Perception of climate change. [Percepción del cambio climático]. Proc. Natl. Acad. Sci. 109, 14726-14727, E2415-E2423, doi:10.1073pnas.12052 76109.

Intergovernmental Panel on Climate Change. (2014). Climate Change 2014: Impacts,
Adaptation and vulnerability. [Cambio climático 2014: impactos, adaptación y vulnerabilidad] Retrieved from https:// www.environment.gov.za/sites/default/ files/docs/impactsadaptation

Jiménez-Vélez, B., Detrés, Y., Armstrong, R. A. $\mathrm{y}$ Gioda, A. (2009). Characterization of African Dust (PM 2.5) across the Atlantic Ocean during AEROSE 2004. [Caracterización del polvo africano (PM 2.5) a través del Océano Atlántico durante AEROSE 2004. Ambiente atmosférico]. Atmospheric Environment 43:2659-2664.

Jury, M. R. (2011). Long-term variability and trends in the Caribbean Sea. [Variabilidad $\mathrm{y}$ tendencias a largo plazo en el Mar Caribe]. International Journal of Oceanography. https://doi.org/10.1155/2011/465810

Meehl, G. A., Tebaldi, C., Walton, G., Easterling, D., y McDaniel, L. (2009). Relative increase of record high maximum temperatures compared to record low minimum temperatures in the US. [Cambio climático, calor y mortalidad en el área urbana tropical de San Juan, Puerto Rico]. Geophysical Research Letters, 36(23). https://doi.org/10.1029/2009GL04073

Méndez-Lázaro, P. A., Pérez-Cardona, C. M., Rodríguez, E., Martínez, O., Taboas, M., Bocanegra, A., \& Méndez-Tejeda, R. (2016). Climate change, heat, and mortality in the tropical urban area of San Juan, Puerto Rico. International Journal of Biometeorology, 1-9. https:// doi.org/10.1007/s00484-016-1291-z

Méndez-Lázaro, P., Martínez-Sánchez, O., Méndez-Tejeda, R., Rodríguez, E., Morales, E., \& Schmitt-Cortijo, N. (2015). Extreme heat events in San Juan Puerto Rico: Trends and variability of unusual hot weather and its possible effects on ecology and society. [Eventos de calor 
extremo en San Juan, Puerto Rico: tendencias y variabilidad del inusual clima caliente y su posible efecto en la ecología y la sociedad. Revista de Climatología y Previsión Meteorológica]. Journal of Climatology \& Weather Forecasting, 1-7. https://doi.org/10.4172/2332-2594 .1000135

Méndez-Tejeda, R., María, S. C., Sergio, O. M., y Oscar, C. V. (2015). Environmental and Economic Impact of Forest Fires in Puerto Rico 2013-2014. [Impacto ambiental y económico en los incendios forestales en Puerto Rico]. Open Journal of Forestry, 5(04), 353. http:// dx.doi.org/10.4236/ojf.2015.54030.

Méndez-Tejeda, R. (2017). Increase in the Number of Hot Days for Decades in Puerto Rico [Aumento en el número de días calurosos por décadas en Puerto Rico]. 19502014. Environment and Natural Resources Research; Vol. 7, No. 3; 2017 ISSN 1927-0488 E-ISSN 1927-0496. doi:10.5539/enrr.v7n3p16

Morelock, J. (1984). Coastal Erosion in Puerto Rico. Shore and Beach: [Erosión costera en Puerto Rico. Costa y Playa]. 18-27. NCA (2014). https://nca2014. globalchange.gov/ NCA (2016). https:// health2016.globalchange.gov/

NWS. ENSO effects across the northeastern Caribbean [Efectos de de ENSO a través del noreste del Caribe]. (2016). http://www.weather.gov/sju/climo_ enso

Ortiz-Royero, J. C., Otero, L. J., Restrepo, J. C., Ruiz, J., y Cadena, M., Cold fronts in the Colombian Caribbean Sea and their relationship to extreme wave events, Nat. [Frentes fríos en el Mar Caribe Colombiano y su rela- ción con los eventos de olas extremas]. Hazards Earth Syst. Sci., 13, 2797-2804, https://doi.org/10.5194/ nhess-13-2797-2013.

Peterson, T. C., et al., Homogeneity adjustments of in situ atmospheric climate data: [Ajustes de homogeneidad de los datos climáticos atmosféricos]. A review, Int. J. Climatol., 18, 1493-1517, 1998 b.

Peterson, T. C., Taylor, M. A., Demeritte, R., Duncombe, D. L., Burton, S., Thompson, F., ... y Klein, T. A.(2002). Recent changes in climate extremes in the $\mathrm{Ca}$ ribbean region. [Cambios recientes en climas extremos en la región del Caribe]. Journal of Geophysical Research: Atmospheres, 107 (D21). https://doi.org/10.1029/2002JD002251PR-CCC.org

Pielke, Jr., R. A., Gratz, J., Landsea, C. W., Collins, D., Saunders, M. A., and Musulin, R., (2008). Normalized Hurricane Damages in the United States: 19002005. [Daños causados por huracanes normalizados en Estados Unidos]. $\mathrm{Na}$ tural Hazards Review, 9, Issue 1, 29-42.

Rhiney, K. (2015). Geographies of Caribbean vulnerability in a changing climate: Issues and trends. [Geografía de la vulnerabilidad caribeña en un clima cambiante: asuntos y tendencias]. Geography Compass, 9(3), 97-114. https:// doi.org/10.1111/gec3.12199.

Rigau-Pérez, J.G., Ayala-López, A., García-Rivera, E.J., Hudson, S.M., Vorndam, V., Reiter, P., Cano, M.P., Clark, G.G., (2002) The reappearance of dengue-3 and a subsequent dengue-4 and dengue-1 epidemic in Puerto Rico in 1998. [La reaparición del dengue -3 y una subsiguiente epidemia de dengue-4 $y$ dengue -1 en Puerto Rico en 1998]. Amer. J. Trop. Med. Hyg., 67, 355-362. 
Schuldt, J. P. (2016). "Global Warming" versus "Climate Change" and the Influence of Labeling on Public Perceptions. Climate Change Communication. ["Calentamiento global" versus "cambio climático" y la etiqueta de la etiqueta sobre la percepción pública. Comunicación del cambio climático]. DOI:10.1093/ acrefore/9780190228620.013.309.

Seguinot, J. (2005): GeoCaribe: Medio ambiente Cultura y Salud en el Caribe Contemporáneo. San Juan, Puerto Rico. Ed. Geo..

Stephenson, T. S. et al (2014). Changes in extreme temperature and precipitation in the Caribbean region, 1961-2010. Int. Journal of Climatol. [Cambios extremos en la temperatura y la precipitación en la región del Caribe]. 34: 29572971 (2014). doi:10.1002/j0c.3889

Tartaglione, C. A., S. R. Smith, y J. J. O’Brien,. (2003). ENSO impact on hurricane landfall probabilities for the Caribbean. [ENSO impacto sobre las probabilidades de recalada del huracán en el Caribe]. J. Climate, 16, 2925-2931, doi:10.1175/1520-0442(2003)016,2925: EIOHLP.2.0.CO;2.

Trenberth, K., Fasullo, J. y Shepherd, T. (2015). Attribution of climate extreme events. [Atribución de eventos climáticos extremos]. Nature Climte Change 5, 725-730. doi:10.1038/nclimate 2657
Union of Concerned Scientists (2017).

https://www.ucsusa.org/global-warming/science-and-impacts/impacts/ hurricanes-and-climate-change.html\#. WlmdNqiWbIU

Van Beusekom, A. E., González, G., y Rivera, M. M. (2015). Short-term precipitation and temperature trends along an elevation gradient in Northeastern Puerto Rico. [Precipitaciones a corto plazo y tendencias de temperatura a lo largo de una gradiente elevación en el noreste de Puerto Rico]. Earth Interactions, 19(3), 1-33. https://doi.org/10.1175/EI-D-140023.1 vulnerability_wg2.pdf

Woosuk, C. et al (2017). Near-Futue prediction of tropical Cyclone Activity over the North Atlantic, [Predicción futura-cercana de la actualidad del ciclón tropical sobre el Atlántico Norte]. Journal of Climate. American Meteorological Society. https://doi. org/10.1175/JCLI-D-17-0206.1

Zhang, W., Vecchi, G., Villarini, G., Murakami, H., Delworth, T., Jia, L., Gudgel, R., y Zeng, F. (2016). Simulated Connections between ENSO and Tropical Cyclones near Guam in a High-Resolution GFDL Coupled Climate Model: Implications for Seasonal Forecasting. [Conexiones simuladas entre ENSO y los ciclones tropicales cerca de Guam en un modelo de clima acoplado GFDL de alta resolución]. Journal of Climate,.29 (22), 8231-8248

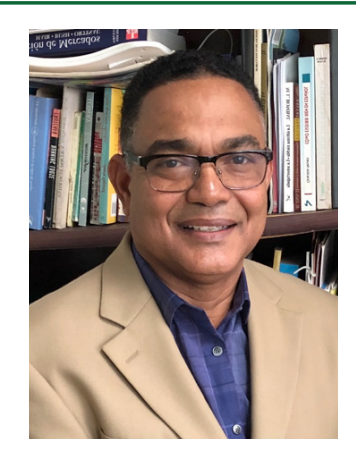

\section{Rafael Méndez Tejeda}

Es catedrático de Física, Meteorología y Climatología de la Universidad de Puerto Rico, en los Recintos de Carolina y Rio Piedras, director del laboratorio de Ciencias Atmosféricas. Tiene más de 50 artículos en "Journals" con pares y diversos artículos de divulgación en revistas y periódicos locales e internacionales. Ha dictado más de 100 conferencias internacionales enAmérica Latina, USAy Europa. Harealizado cientos de entrevistas deradio y televisión. Además, es parte del "Editorial Board" de varias revistas entre ellas Journal of Geography and Geology (http://www.ccsenet.org/journal/index.php/jgg) y “reviewer” del Journal in Geoscience and Environmental Protection (http://www.scirp.org/journal/gep/) 